\title{
Comparison of Cholinergic Status with Quantitative EEG in Healthy Subjects and Patients Suspected of Dementia
}

\author{
Rolf Ekedahl* \\ Department of Neurophysiology, Sweden
}

*Corresponding author: Rolf Ekedahl, Department of Neurophysiology, Neufydi AB, Sweden.
Received Date: April 07, 2019

Published Date: April 12, 2019

\section{Abstract}

Introduction: Objective is to evaluate quantitative electroencephalography (qEEG) variables to distinguish healthy subjects from patients who investigated for dementia and, to assess also at follow-up examination if acetylcholinesterase inhibitor (AChEI) treatment or not influenced the qEEG.

Methods: Average value from four EEG epochs obtained with eyes closed (E.Cl.) and eyes open (E.O.), the peak frequency with eyes closed and the Vigilance-index (ratio of E.0./E.Cl. average powers) calculated. A healthy group and a group suspected of having primary dementia compared, and the assumed dementia group evaluated with follow-up qEEG after approximately 12-14 months with or without acetylcholinesterase inhibitor treatment. The statistical analyze (Student`s t-test) p-values compared the healthy individuals against the suspected dementia patients, and at follow-up of AChEI treated against non-treated analyzed with paired p-values.

Result: When the healthy group compared with suspected dementia group at baseline, all variables were altered statistically significant except E.Cl. average power. The Vigilance-index (p-value $<0.001$ ) and average power of E.O. (p-value $<0.001$ ) increased, and the mean peak- frequency ( $p$-value $<0.001$ ) decreased. At the follow-up for the suspected dementia group, the Vigilance-index of the untreated increased significantly ( $p$-value $<0.001$ ) but not for the AChEI treated group. No other statistically significant changes for the other parameters.

Conclusion: The Vigilance-index may be used to assess cholinergic deficits in patients with dementia, also early in the course of the disease and evaluate the effects of AChEI treatment. Also, identify unwanted central cholinergic side-effects of other medication.

Keywords: Electroencephalography; Desynchronization; Dementia; Acetylcholine; Cholinesterase inhibitors, Alzheimer's dementia; Lewy body disease; Humans; Biomarkers; Follow-up studies

\section{Introduction}

Longitudinal studies using electroencephalography (EEG) and follow-up are rare in dementia studies; however, varying effects on EEG parameters reported from existing studies [1-7]. Probably due to the heterogeneity and different stages of the dementia diseases, and also mixed types of dementia, e.g., Alzheimer's dementia (AD), vascular dementia (VaD), Lewy body dementia (LBD) studied. Interpreting the EEG measurements is also tricky because the procedures used to collect and analyze data are different. The commonly used EEG parameters have assessed changes in the proportion of different frequency bands, average frequency, EEG power, the ratio of low-frequency activity to global and fluctuations in EEG frequency and coherence.

Varying proportions of patients did not display EEG changes at follow-up compared to baseline values $[1,3,4,5,8]$. Therefore, examinations of other EEG parameters that better reflect the electrophysiological changes in patients with dementia diseases needed for improving the diagnostic precision. Particularly in the early stages of dementia, and to monitor disease progression and evaluate the effects of Acetylcholinesterase inhibitor (AchEI) treatment. One possible way is to try to find methods to measure the cholinergic deficit in patients with primary degenerative dementia, such as AD [9-17]. Which, potentially could lead to an earlier AChEI treatment initiation in the early course of the disease than previous EEG methods [18-28] and other diagnostic measures, but also be used as a biomarker for dementia disease characterized by cholinergic deficiency.

Most of previous EEG studies about cholinergic changes and EEG power effects, observed when cholinergic and anticholinergic 
drugs been tested with a decrease in relative power for Alfa-activity (8-13 Hz) of global power and increased power at eye-opening when anticholinergic drugs tested [19,22,29-34]. Consequently, a new measurement introduced in this study, the ratio of E.O/E.Cl. average power termed Vigilance-index, which measures the relative reactivity at eye-opening in relation to eyes closed resting activity (desynchronization) and therefore quantifies Berger reactivity [35], with the potential to estimate the cholinergic status.

In this study, the objective is to identify EEG variables at baseline that could separate patients suspected of having dementia and healthy subjects. For the suspected dementia group, identify EEG variables useful to trace the possible influence of AChEI treatment on the cholinergic deficit, by evaluating patients treated or not treated with AChEI after baseline at follow-up examination.

\section{Materials and Methods}

\section{Materials}

Two groups compared and analyzed, healthy subjects as control group and patients suspected of dementia which was investigated at a geriatric clinic. The suspected dementia group divided into those with no AChEI treatment (48 patients average of age $78.8 \pm 6.5$ years) and patients who received AChEI treatment (18 patients average of age $78.3 \pm 5.7$ years) between the baseline and follow-up EEG. Selected patients were in an early stage of a dementia disease, or the dementia diagnosis was uncertain. Thirty healthy volunteers who were the control group had an average age of $42.9 \pm 11.9$ years and not treated with anticholinergic or other cholinergic medication, and no drug/alcohol addiction reported or suspected. The ethical committee at Karolinska Institute approved analyses, and informed consent obtained from the healthy subjects and patients.

\section{EEG recordings and analysis}

EEGs recorded from awake patients and subjects who were sitting on regular chairs and instructed to open or close their eyes on command every 90 seconds which ensured alertness and vigilance. Alertness also monitored by the EEG assistant during the recording, that lasted for approximately 11 minutes. The patients and parts of the EEG recordings that showed signs of drowsiness were excluded from the analysis. Patients and healthy subjects who were very tense or nervous and had low EEG amplitudes during eye-closure, comparable to the magnitudes at eye-opening [36], were also excluded from the analyses. Five patients and three healthy subjects were excluded from the analysis of this reason.

EEGs were recorded and analyzed using standard digital EEG equipment (Nervus ${ }^{\mathrm{TM}} 5.3$ digital EEG system, Viasys Healthcare, Inc., San Diego, California, United States). The recorded analog signal converted to a digital signal by Fast Fourier transformation (FFT) with a sampling frequency of $128 \mathrm{~Hz}$. At least four EEG epochs, of 90 seconds with eyes closed and 30 seconds with eyes opened recorded. Approximately eighty seconds of each eye closed epoch and twenty-five seconds with eyes open used in the analysis. The first 10 seconds were excluded from eyes closed epochs to avoid analyses of arousal and movement artifacts and the first 3 seconds of eyes open epochs similarly excluded. Rarely slightly shorter epochs analyzed due to recording interference.

The average peak power for four eye closure epochs and open eye epochs, within $\pm 1 \mathrm{~Hz}$ range of average peak-frequency with eyes closed, calculated. Vigilance-index decided from the ratio of the average peak power for open eye epochs and for closed eyes epochs (E.O. power/E.Cl. power) and quantified relative desynchronization of average peak power. The peak-frequency and power measurements defined within EEG filter setting of 3-15 $\mathrm{Hz}$, which covered alpha and theta frequency bands (4-13 Hz) and avoided analysis of eye movement and muscle artifacts. EEGs analyzed from the T6 or T5 area (10-20 EEG system) covering posterior part of the temporal lobe, which is affected by Alzheimer`s and Lewy body dementia and an area of Cortex that alfa-rhythm are prominent.

When comparing Vigilance-index, MMSE and Cerebrospinal fluid (Csf) content of T-Tau, phospho-Tau and amyloid-42, a quota calculated, of the number of patients with normal value for MMSE score or for (Csf) content used in clinical practice as the denominator and the number of patients with pathological MMSE scores or (Csf) content as the numerator.

\section{Statistical analysis}

The average peak-frequencies, peak-power, the standard deviation (SD) and the standard error of the mean (S.E.M.) for the compared groups calculated, and differences between the mean values in the healthy and suspected dementia groups estimated using p-values with variance and two-tailed Student's t-tests. Paired two-tailed Student's t-tests p-values used to compare AchEI treated and untreated patient groups at follow-up EEG and MMSE examinations.

\section{Result and Discussion}

When the healthy group compared with suspected dementia group at baseline, all variables were altered significantly except E.Cl. average power. The Vigilance-index (p-value $<0.001$ ) and average power of E.O. ( $p$-value < 0.001) increased, and the mean peakfrequency ( $p$-value $<0.001$ ) decreased for the suspected dementia group Table 1. The mean standard deviations (SD) and standard error of the mean (S.E.M.) for the healthy subjects: E.Cl. average power (16.2 SD 15.15 S.E.M.: 2.86), E.O. average power (1.8 SD 1.75 S.E.M.: 0.33), average peak-frequency (10.0 SD 0.73 S.E.M.: 0.14) and Vigilance-index (0,19 SD 0.21 S.E.M.: 0.04). For the suspected dementia patients at baseline: E.Cl. (12.5 SD 16.4 S.E.M.:2.02), E.O. (3.9 SD 4.2 S.E.M.: 0.52), average peak-frequency (8.6 SD 1.02 S.E.M.: 0.12) and Vigilance-index (0.5 SD 0.34 S.E.M.:0.04) (Table 1).

Therefore Vigilance-index seems most appropriate EEG variable to distinguish healthy subjects from suspected dementia patients. However, there is no defined normal value for Vigilanceindex, but the comparison between the average value and SD for the healthy subjects $(0.2$ SD 0,21$)$ and the suspected dementia group (0.5 SD 0.34) leads to my suggestion to use $<0.3$ as reasonable normal value for Vigilance-index (Figure 1). 
A

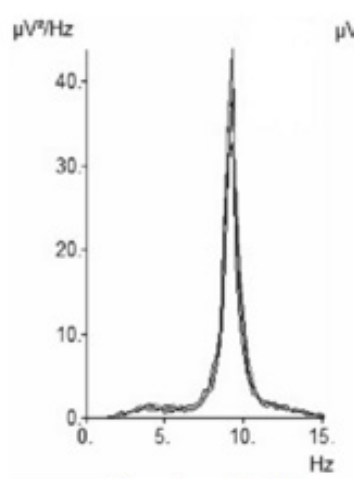

Eyes closed (E.Cl.)
B
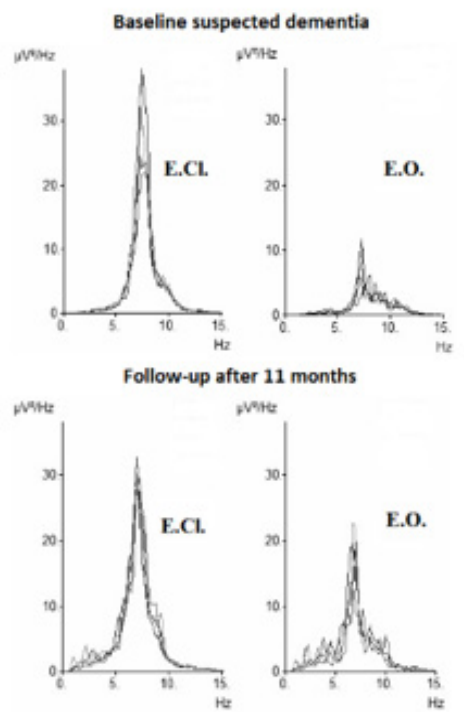

Figure 1: Quantitative EEG-analysis for a healthy person and a suspected dementia patient at baseline and follow-up. In all diagrams, the $\mathrm{X}$-axis shows the frequency $(\mathrm{Hz})$, and the $\mathrm{Y}$-axis shows the power $\left(\mu \mathrm{V}^{2} / \mathrm{Hz}\right)$, in graph four EEG epochs superimposed showed. A: (left), four epochs superimposed when eyes are closed (E.Cl.) and to the right when eyes are open (E.O.) for a healthy person. B: Comparison with a patient suspected to have dementia without AChEI treatment, at baseline (upper diagram) and lower diagram follow-up examination after 11 months. Observe that power and frequency decreased at the follow-up EEG for the E.Cl. condition, and EEG power substantially increased for E.O. condition compared to baseline for the suspected dementia patient.

Table 1: Statistical analyses ( $p$-value) of the examined EEG variables (upper row). The different comparisons are shown in the left column. Second row: all variables but E.CL. average power showed significant differences between the suspected dementia patients compared to healthy subjects at baseline EEG. Third row: Differences between baseline and follow-up EEGs after 14 months (SEM: 2.3) of patients with suspected dementia and not Acetylcholinesterase inhibitor (AChEI) treated. Fourth row: Differences between baseline and follow-up EEGs after 12 months (SEM: 1.65) for AChEI treated between baseline and follow-up EEG. Observe the significant difference for Vigilance-index (bold italics).

\begin{tabular}{|c|c|c|c|c|}
\hline $\begin{array}{c}\text { Student's t-test } \mathbf{p} \text {-value } \mathbf{p}< \\
\mathbf{0 . 0 5}=\boldsymbol{*}_{\mathbf{p}} \mathbf{<} \mathbf{0 . 0 1}=* *, \mathbf{p}<\mathbf{0 . 0 0 1} \\
\mathbf{=} * * *\end{array}$ & $\begin{array}{c}\text { E.Cl. (Eyes closed) power } \\
\text { average value }\end{array}$ & $\begin{array}{c}\text { E.0. (Eyes open) power } \\
\text { average value }\end{array}$ & Vigilance-index E.0./E.Cl. & $\begin{array}{c}\text { Peak-frequency average } \\
\text { value }\end{array}$ \\
\hline $\begin{array}{c}\text { Healthy subjects / patients with } \\
\text { suspected dementia at baseline }\end{array}$ & 0.318 & $0.0007^{* * *}$ & $9.3 \times 10^{-} 8^{* * *}$ & $2.7 \times 10^{-} 10^{* * *}$ \\
\hline Untreated Baseline / follow-up & 0.742 & 0.051 & $0.00082 * * *$ & 0.157 \\
\hline Treated Baseline / follow-up & 0.661 & 0.974 & 0.932 & 0.164 \\
\hline
\end{tabular}

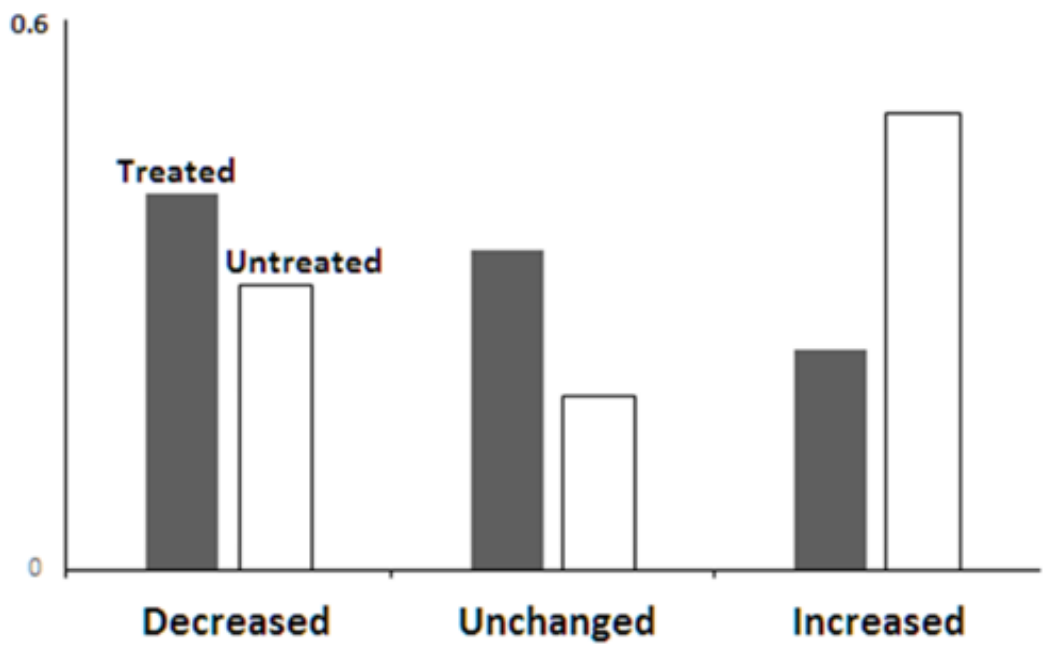

Figure 2: Comparison of the Vigilance-index at the follow-up EEG of patients who were treated with or without AChEl after the baseline EEG. Categories on the X-axis include (from left to right): decreased, unchanged and increased Vigilance-index at follow-up EEG. The Y-axis shows the relative proportion of treated or untreated patients in different categories. The treated group is shown as filled and the untreated group as unfilled bars. Observe that the treated group had a higher proportion of patients with decreased or unchanged Vigilance-index and fewer patients with an increased Vigilance-index than the untreated patients. 
To measure the cholinergic status and efficacy of the AchEI treatment, a paired statistical comparison of the Vigilance-index for the AchEI treated and the non-treated group between baseline and follow-up performed. The untreated group demonstrated a significant increase of Vigilance-index $(p<0.001)$ while the treated group did not $(p=0.932)$. The other EEG variables: average E.CL., and E.O. power as well as average peak- frequency did not change significantly for the treated nor the untreated groups Table 1 . There were different changes of the Vigilance-index for both groups of patients, with decreasing, unchanged or increased Vigilance-index. A more substantial proportion of the AchEI treated patients than the untreated had a reduced or unchanged Vigilance-index at followup. However, the most substantial difference was the proportion of patients with increased Vigilance-index, relatively in approximately twice as many of the untreated compared to the treated patients (Figure 2).

Which differences characterized the groups selected for AchEI treatment compared to the untreated group at baseline? The treated group had higher mean Vigilance-index (0.6) and lower MMSE (average score 24,9) than the untreated (0.5) respectively $(25,9)$. Csf data paradoxically showed a higher number of pathological Amyloid 42 for the untreated compared to the treated group (relative proportions of 0.25 respectively 0.1 ), and lower number for total-tau (0.39 respectively 0.5$)$ and phospho-tau $(0.17$ respectively 0 ) (Table 2).

Table 2: Comparison between healthy subjects and suspected dementia patients, with or without AchEl treatment. In the first upper row, the biomarkers compared. For Csf biomarkers only baseline-values examined. The first column from left, the groups of suspected dementia patients, AchEl treated or not treated examined at baseline and follow-up. For Vigilance-index and MMSE score, average values with S.E.M. and number of patients in italic text for the examined. For the Csf data a quota of pathologic value / total value for respectively phospho-Tau, total-Tau, and Amyloid- $\beta^{42}$ specified.

\begin{tabular}{|c|c|c|c|c|c|}
\hline & $\begin{array}{c}\text { Vigilance-index average } \\
\text { value }\end{array}$ & $\begin{array}{c}\text { MMSE score average } \\
\text { value }\end{array}$ & $\begin{array}{c}\text { phospho-Tau quota } \\
\text { pathologic value }\end{array}$ & $\begin{array}{c}\text { total-Tau quota } \\
\text { pathologic value }\end{array}$ & $\begin{array}{c}\text { Amyloid-b42quota } \\
\text { pathologic value }\end{array}$ \\
\hline Baseline not treated & $\begin{array}{c}0.5 \text { (S.E.M. 0.050) } 48 \\
\text { patients }\end{array}$ & $\begin{array}{c}25,9 \text { (S.E.M. 0.563) } 35 \\
\text { patients }\end{array}$ & 0.1733 patients & 0.39 & 0.25 \\
\hline Follow up not treated & 0.6 (S.E.M. 0.052) & 25,3 (S.E.M. 0.579) & & 0.5 & 0.1 \\
\hline Baseline treated & $\begin{array}{c}0.6 \text { (S.E.M. 0.082) } 18 \\
\text { patients }\end{array}$ & $\begin{array}{c}24,9 \text { (S.E.M. 1.129) } 15 \\
\text { patients }\end{array}$ & 010 patients & & \\
\hline Follow up treated & 0.6 (S.E.M. 0.085) & 22,9 (S.E.M. 0.879) & & & \\
\hline
\end{tabular}

The decreasing E.Cl. power and increasing E.O. power in patients with suspected dementia and consequently increased Vigilance-index affected by AchEI treatment are consistent with pharmacological studies of the effects of cholinergic and anticholinergic substances [18-19,22-23,29-31,34] and AChEI drugs [21,24,37-39] on EEG variables. Reductions of desynchronization when eyes are open or Berger reactivity has also been observed following the administration of anticholinergic drugs, such as Atropine and Scopolamine, to healthy volunteers [22, 29]. Comparison between healthy subjects and patients with AD, revealed significantly reduced desynchronization reaction at eyeopening in patients with $\mathrm{AD}$ [33].

The observed decrease in the average peak-frequency in suspected dementia patients is also consistent with previous longitudinal studies of patients with dementia [3,5-6,34] and other studies related to EEG changes in patients with dementia, which also reported a decreasing power for alpha activity [25,40-47].

These EEG-parameters should best distinguish between healthy and early dementia. However, there are no normal values for average E.Cl. and E.O. power due to considerable variations between individuals and high SD for these variables and overlap between average value for healthy subjects and suspected dementia group and therefore not suitable for early dementia diagnostics. The Vigilance-index have the advantage to erase the substantial differences of EEG power between individuals and makes a comparison possible due to a quota of powers. The average peakfrequency, even though it could distinguish healthy from dementia suspected statistical significant, it still was within normal values $(8.6 \mathrm{~Hz})$ and of that reason not suitable for early dementia diagnosis.
Increased Vigilance-index depends on a decrease in ECl. power and especially of increase in E.O. power, which was significant in this study ( $p$-value $<0.001$ ) when normal subjects compared to patients with suspected dementia. The Vigilance-index at the follow-up EEG increased to a significant level ( $p$-value $<0.001$ ) in the not treated patients, while statistically unchanged in the AChEI treated group, supporting the notion that the cholinergic status was measured. As fewer AChEI treated patients had increased, and several unchanged or decreased Vigilance-index than the untreated patients Figure 2, Vigilance-index might perhaps distinguish responders from non-responders when AchEI treatment instituted. Patients with an increasing index seem not to react to AchEI treatment, while those with a decreasing or unchanged index might indicate a treatment effect with increased or constant availability of Acetylcholine. The group treated with AchEI was restricted (18 patients), and these observations must be supplemented with investigations of additional patients and longer observation time before the Vigilance-index definitively used to determine treatment effects.

Were the different results for the studied groups dependent on group differences? The criteria for either treatment or not with AchEI were based on the assessment of the patient's physician. The treated group had lower average MMSE scores at baseline and higher Vigilance-index than the untreated group, so it seems that the treated had more positive symptoms of dementia Table 2.

A puzzling observation, when Csf could be compared for the treated and untreated group, Amyloid- $\beta^{42}$ was pathologic for $1 / 10$, phospho-Tau for $0 / 10$ and total-Tau for $5 / 10$ for the treated patients, and the untreated; $8 / 33$ was pathologic for 
Amyloid- $\beta^{42}, 13 / 33$ for total-Tau and 6/33 for phospho-Tau Table 2. Csf biomarkers seem to be a poor indicator in the early stage of suspected dementia. However, this needs to be studied in larger groups of patients.

Other studies have attempted to develop EEG methods for estimating the cholinergic status in patients with dementia diseases, these methods based on algorithms of analyzing EEG-signals from several electrodes and different aspects of electrical changes in EEG [26-28]. The use of several electrodes and variables increases the possible sources of error, which is a flaw for these methods. The strategy in this study, to only use two variables and analyzing the ratio between the quota of E.Cl. and E.O. average power erases the individual differences in EEG power which makes it possible to compare different individuals and compare the same patient at separate occasions. By analyzing one EEG channel in the posterior part of the temporal region, makes the Vigilance-index robust, less sensitive to EEG artifacts and easy to use clinically.

\section{Conclusion}

The Vigilance-index assess the cholinergic status in the posterior part of the temporal lobe of the Cortex and is potentially useful for the diagnosis of primary dementia which is characterized by a deficiency in acetylcholine, such as in Alzheimer's disease and Lewy body dementia. The Vigilance-index allows physicians to detect dementia early and evaluate acetylcholine deficiency. Besides, the Vigilance-index might also possibly used to evaluate Acetylcholinesterase inhibitor treatment, aimed to increase the availability of acetylcholine and thereby identify responders versus non-responders and also identify other medications with central anticholinergic side-effects.

\section{Acknowledgment}

The author thanks Dr. Stig Samuelsson for recruiting patients from the Geriatric outpatient unit at Nacka hospital in Sweden, to this study. The author also wants to thank the staff at the same unit, notably Nina Stark, for her assistance with the EEG-recording.

\section{Conflict of Interest}

This research was not funded by any specific grant from funding agencies in the public, commercial, or not-for-profit sectors. Nonconflict of interest exists.

\section{References}

1. Gordon E (1968) Serial EEG studies in presenile dementia. Br J Psychiatry 114(511): 779-780.

2. Johannesson G, Brun A, Gustafson L, Ingvar D (1977) EEG in presenile dementia related to cerebral flow and autopsy findings. Acta Neurol Scand 56(2): 89-103.

3. Coben L, Danzinger W, Storandt M (1985) A longitudinal EEG study of mild senile dementia of Alzheimer type: changes at 1 year and at 2.5 years. Electroencephalogr Clin Neurophysiol 61(2): 101-120.

4. Rae Grant A, Blume W, Lau C, Hachinski V, Fisman M, et al. (1987) The electroencephalogram in Alzheimer-type dementia. A sequential study correlating the electroencephalogram with psychometric and quantitative pathologic data. Arch Neurol 44(1): 50-54.

5. Soininen H, Partanen J, Laulumaa V, Pääkönen A, Helkala E L, et al. (1991) Serial EEG in Alzheimer's disease: 3 year follow-up and clinical outcome. Electroencephalogr Clin Neurophysiol. 79(5): 342-348.
6. Jelic V, Johansson SE, Almkvist O, Shigeta M, Julin P, et al. (2000) Quantitative electroencephalography in mild cognitive impairment: longitudinal changes and possible predictions of Alzheimer's disease. Neurobiol Aging 21(4): 533-540.

7. Bonanni L, Thomas A, Tiraboschi P, Perfetti B, Varanese S (2008) EEG comparison in early Alzheimer's disease, dementia with Lewy bodies and Parkinson's disease with dementia patients with a 2-year follow-up. Brain 131(pt 3): 690-705.

8. LetemendiaF,Pampiglione G (1958)Clinicaland Electroencephalographic observations in Alzheimer's disease. J Neurol Neurosurg Psychiatry (21): 167-172.

9. Drachman D, Leavitt J (1974) Human memory and the cholinergic system. A relationship to aging? Arch Neurol 30(2): 113-121.

10. Davies P, Maloney AJ (1976) Selective loss of central Cholinergic neurones in Alzheimer's disease. Lancet 2(8000): 1403.

11. Perry E, Tomlinson B, Blessed G, Bergman K, Gibson P, et al. (1978) Correlation of cholinergic abnormalities with senile plaques and mental test scores in senile dementia. Br Med J 2(6150): 1457-1459.

12. Bartus R, Dean R, Beer B, Lippa A (1982) The cholinergic hypothesis of geriatric memory dysfunction. Science 217(4558): 408-414.

13. Coyle J, Price D, DeLong M (1983) Alzheimer's Disease: A Disorder of Cortical Cholinergic Innervation. Science 219(4589): 1184-1190.

14. Francis P, Palmer A, Sims N, Bowden D, Davison A, et al. (1985) Neurochemical studies of early-onset Alzheimer's disease. Possible influence on treatment. N Engl J Med 313(1): 7-11.

15. Frölich L (2002) The cholinergic pathology in Alzheimer's diseasediscrepancies between clinical experience and pathophysiological findings. J Neural Transm 109(7-8): 1003-1014.

16. Terry A, Buccafusco J (2003) The Cholinergic Hypothesis of Age and Alzheimer's Disease-Related Cognitive Deficits: Recent Challenges and Their implications for Novel Drug Development. J Pharmacol Exp Ther 306(3): 821-827.

17. Martorana A, Esposito Z, Koch G (2010) Beyond the Cholinergic Hypothesis: Do Current Drugs Work on Alzheimer's Disease. CNS Neuroscience \& Therapeutics 16(4): 235-245.

18. Agnoli A, Martucci N, Manna V, Conti L, Fioravanti M (1983) Effect of Cholinergic and anticholinergic drugs on short term memory in Alzheimer's dementia: a computerized Electroencephalographic study. Clin Neuropharmacol. 6(4): 311-323.

19. Sannita W, Maggio L, Rosadino G (1987) Effects of Scopolamine (0.25$0.75 \mathrm{mg}$ i.m.) on the Quantitative EEG and the Neuropsychological status of healthy volunteers. Neuropsychobiology 17(4): 199-205.

20. PJ Riekkinen, PJ Riekkinen, H Soininen, KJ Reinikainen, V Laulumaa, et al. (1990) Regulation of EEG delta activity by the cholinergic nucleus basalis. In: Maurer K, Riederer P, Beckmann H, (Eds.), Alzheimer's Disease Epidemiology, Neuropathology, Neurochemistry, and Clinics Key Topics in Brain Research. Springer Verlag, Vienna, Austria, pp. 437-445.

21. Alhainen K, Partanen J, Reiniainen K, Laulumaa V, Soininen H, et al. (1991) Discrimination of Tetrahydoaminoacridine responders by a single dose pharmaco-EEG in patients with Alzheimer's disease. Neurosci Lett 127(1): 113-116.

22. Sloan E, Fenton G, Standage K (1992) Anticholinergic drug effects on quantitative Electroencephalogram, visual evoked potentials and verbal memory. Biol Psychiatry 31(6): 600-606.

23. Neufeld M, Rabey M, Parmet Y, Sifris P, Treves T, et al. (1994) Effects of single intravenous dose of scopolamine on the quantitative EEG in Alzheimer's disease patients and age-matched controls. Electroencephalogr Clin Neurophysiol 91(6): 407-412.

24. Reeves R, Struve F, Patrick G (2002) The Effects of Donepezil on Quantitative EEG in Patients with Alzheimer's Disease. Clin Electroencephalogr 33(2): 93-96.

25. Jeong J (2004) EEG dynamics in patients with Alzheimer's disease. J Clin Neurophysiol 115(7): 1490-1505. 
26. Babilonia C, Frisonic G, Vecchioc F, Pievani M, Geraldi C, et al. (2010) Global Functional Coupling of resting EEG Rythms is Related to WhiteMatter Lesions Along the Cholinergic Tracts in Subjects with Amnesic Mild Cognitive Impairment. Journal of Alzheimer's Disease 19(3): 859871.

27. Snaedal J, Johannesson G, Gudmundsson T, Emilsdottir N, Einarsson B, et al. (2012) Diagnostic Accuracy of Statistical Pattern Recognition of Electroencephalogram Registration in Evaluation of Cognitive Impairment and Dementia. Dement Geriatr Cogn Disord 34(1): 51-60.

28. Johannsson M, Snaedal J, Johannesson G, Gudmundsson T, Johnsen K (2015) The Acetylcholine Index: An Electroencephalographic Marker of Cholinergic Activity in the Living Human Brain Applied to Alzheimer's Disease and Other Dementias. Dement Geriatric Cogn Disord 39(3-4): 132-142.

29. White R, Rinaldi F, Himmich H (1956) Central and peripheral nervous effect of Atropine sulfate and Mepiperphenidol bromide (Darstine) on human subjects. J Appl Physiol 8: 635-642.

30. Martin W, Eades C (1960) A Comparative Study of the Effect of Drugs on Activating and Vasomotor Responses Evoked by Mid Brain Stimulation: Atropine, Pentobarbital, Chlorpromazine and Chlorpromazine Sulfoxide. Psychopharmacology 1(4):303-335.

31. Kanak T, Szerb J (1965) Mesencephalic reticular activating system and cortical acetylcholine output. Nature 205: 80-82.

32. Sie G, Jasper H, Wolfe I (1965) Rate of release from cortical surface in encephale and cerveau isolé preparations in relation to arousal and epileptic activation of the EEG. Electroencephalogr Clin Neurophysiol. 18: 206.

33. Hiele Kvd, Bollen E, Vein A, Reijntjes R, Westendorp R, et al. (2008) EEG markers of future cognitive performance in the elderly. J Clin Neurophysiol 25(2): 83-89.

34. Longo W (1966) Behavorial and Electroencephalographic effects of Atropine and related compounds. Pharmacol Rev 18(2): 965-996.

35. Berger H (1933) Über das Elektrenkephalogramm des Menschen. Archive f Psychiatrie 99: 555-574.

36. Niedermeyer E (2007) The normal EEG of the waking adult. In: Niedermeyer E, Silva FLd, (Eds.), Electroencephalography. ( $5^{\text {th }}$ edn.), Lippincott, Williams \& Wilkins, Philadelphia, USA, pp 167-192.
37. Holl G, Straschill M, Thomsen T, Fischer J, Kewitz H (1992) Effect of the cholinesterase inhibiting substance Galanthamine on Human EEG and visual evoked potentials. Electroencephalogr Clin Neurophysiol. 82(6): 445-452.

38. Babiloni C, Casseta F, Forno GD, Percio CD, Ferreri F, et al. (2006) Donepezil effects on sources of cortical rhythms in mild Alzheimer's disease: Responders vs. Non-Responders. Neuroimage (31(4):16501665

39. Babiloni C, Percio CD, Bordet R, Bourriez JL, Bentivoglio M, et al. (2013) Effects of Acetylcholinesterase inhibitors and Memantine on restingstate electroencephalographic rhythms in Alzheimer's disease patients. J Clin Neurophysiol 124(5): 837-850.

40. Stigsby B, Johannesson G, Ingvar D (1981) Regional EEG analysis and regional cerebral blood flow in Alzheimer's and Pick's disease. Electroencephalogr Clin Neurophysiol 51(5): 537-547.

41. Soininen H, Partanen V, Helkala EL, Riekkinen P (1982) EEG findings in senile dementia and normal aging. Acta Neurol Scand 65(1): 59-70.

42. Pentillä M, Partanen V, Soininen H, Riekkinen P (1985) Quantitative analysis of Occipital EEG in different stages of Alzheimer's disease. Electroencephalogr Clin Neurophysiol 60(1): 1-6.

43. Brenner R, Ulrich R, Spiker D, Sclabassi R, Reynolds C, et al. (1986) Computerized spectral analysis in eldery normal, demented and depressed subjects. Electroencephalogr Clin Neurophysiol 64(6): 483492.

44. Filipovic S, Gueguen B, Derouesne C, Ancri D, Bourdel MC, et al. (1989) Dementia of the Alzheimer type: some features of the posterior cerebral electric activity. Psychiatry Res 29(3): 409-410.

45. Primavera A, Novello P, Finocchi C, Canevori E, Costello L (1990) Correlation between Mini-mental state examination and Quantitative electroencephalography in senile dementia of Alzheimer type. Neuropsychobiology 23(2): 74-78.

46. Schreiter Gasser U, Gasser T, Ziegler P (1993) Quantitative EEG analysis in early onset Alzheimer's disease: a controlled study. Electroencephalogr Clin Neurophysiol 86(1): 15-22.

47. Jelic V, Shigeta M, Julin P, Almkvist O, Winblad B, et al. (1996) Quantitative electroencephalography power and coherence in Alzheimer's disease and mild cognitive impairment. Dement Geriatr Cogn Disord. 7(6): 314323. 